\title{
SPATIALLY HOMOGENEOUS BOLTZMANN EQUATION FOR RELATIVISTIC PARTICLES*
}

\author{
ROBERT M. STRAIN ${ }^{\dagger}$ AND SEOK-BAE YUN ${ }^{\ddagger}$
}

\begin{abstract}
The spatially homogeneous Boltzmann equation has been studied extensively in the Newtonian case, but not much is known for the special relativistic case. In this paper, we address several issues for the spatially homogeneous Boltzmann equation for relativistic particles. We first derive the relativistic version of the Povzner inequality. Using this, we study the Cauchy problem and investigate how the polynomial and exponential moments in $L^{1}$ are propagated. Several key differences between the relativistic and the Newtonian cases are confronted and discussed.
\end{abstract}

Key words. Boltzmann equation, kinetic theory of gases, special relativity, Povzner inequality, moment estimates

AMS subject classifications. 35Q20, 76P05, 83A05, 82B40

DOI. $10.1137 / 130923531$

1. Introduction. The special relativistic Boltzmann equation describes the evolution of the statistical distribution of gaseous particles in the Minkowski space time $[16,18]$ :

$$
\partial_{t} g+\hat{p} \cdot \nabla_{x} g=Q(g, g)
$$

Here $g(x, p, t)$ is the velocity distribution function representing the number density of particles at the point $(x, p)$ in $\mathbb{R}_{x}^{3} \times \mathbb{R}_{p}^{3}$ at time $t \in \mathbb{R}_{+}$. The collision operator $Q$ captures the effect of the collisions or interactions between the particles. We divide it into the gain term $Q^{+}$and the loss term $Q^{-}$as

$$
Q(f, g)(p)=Q^{+}(f, g)(p)-Q^{-}(f, g)(p),
$$

where $Q^{+}$and $Q^{-}$take the following form:

$$
\begin{aligned}
& Q^{+}(f, g)(p)=\frac{1}{p^{0}} \int_{\mathbb{R}^{3}} \frac{d q}{q^{0}} \int_{\mathbb{R}^{3}} \frac{d p^{\prime}}{p^{\prime 0}} \int_{\mathbb{R}^{3}} \frac{d q^{\prime}}{q^{\prime 0}} W\left(p, q \mid p^{\prime}, q^{\prime}\right) f\left(p^{\prime}\right) g\left(q^{\prime}\right), \\
& Q^{-}(f, g)(p)=\frac{1}{p^{0}} \int_{\mathbb{R}^{3}} \frac{d q}{q^{0}} \int_{\mathbb{R}^{3}} \frac{d p^{\prime}}{p^{\prime 0}} \int_{\mathbb{R}^{3}} \frac{d q^{\prime}}{q^{\prime 0}} W\left(p, q \mid p^{\prime}, q^{\prime}\right) f(p) g(q) .
\end{aligned}
$$

Before we proceed, we need to define some terms and notions. The four-momentum $p^{\mu}(\mu=0,1,2,3)$ is defined by $p^{\mu}=\left(p^{0}, p\right)$, where $p^{0}=\sqrt{1+|p|^{2}}$ and $p=\left(p_{1}, p_{2}, p_{3}\right)$ denote the energy and the momentum of a particle respectively. We use $|\cdot|$ to denote the Euclidean norm: $|p|^{2}=\left(p_{1}^{2}+p_{2}^{2}+p_{3}^{2}\right)^{1 / 2}$. Also, we have set the mass of the particle and the speed of light into unity for simplicity. The normalized velocity $\hat{p}$ is defined by $\hat{p}=p / \sqrt{1+|p|^{2}}$. We adopt the signature of metric $(-,+,+,+)$ and define

${ }^{*}$ Received by the editors June 4, 2013; accepted for publication (in revised form) November 11, 2013; published electronically February 20, 2014.

http://www.siam.org/journals/sima/46-1/92353.html

${ }^{\dagger}$ Department of Mathematics, University of Pennsylvania, Philadelphia, PA 19104-6395 (strain@ math.upenn.edu). This author was partially supported by NSF grants DMS-1200747 and DMS-0901463 and by an Alfred P. Sloan Foundation research fellowship.

${ }^{\ddagger}$ Department of Mathematics, Sungkyunkwan University, Suwon 440-746, Republic of Korea (sbyun01@skku.edu). 
the Lorentz inner product by $p^{\mu} q_{\mu}=-p^{0} q^{0}+p \cdot q$, where $p \cdot q$ is the usual Euclidean inner product. We note that $p^{\mu}$ satisfies the mass shell condition: $p^{\mu} p_{\mu}=-1$.

The transition rate $W$ is given by the product of the differential cross section $\sigma(\varrho, \theta)$ and the Dirac delta pertaining to the information of the local conservation laws between two interacting particles:

$$
W\left(p, q \mid p^{\prime}, q^{\prime}\right)=s \sigma(\varrho, \theta) \delta^{(3+1)}\left(p^{\mu}+q^{\mu}-p^{\prime \mu}-q^{\prime \mu}\right) .
$$

Here $\delta^{(3+1)}$ is the four-dimensional Dirac delta function, $s$ denotes the square of the energy in the center of momentum system $p+q=0$,

$$
\begin{aligned}
s & =-\left(p^{\mu}+q^{\mu}\right)\left(p_{\mu}+q_{\mu}\right) \\
& =2\left(p^{0} q^{0}-p \cdot q+1\right) \geq 0,
\end{aligned}
$$

and $\varrho$ is the relative momentum

$$
\begin{aligned}
\varrho & =\sqrt{\left(p^{\mu}-q^{\mu}\right)\left(p_{\mu}-q_{\mu}\right)} \\
& =\sqrt{2\left(p^{0} q^{0}-p \cdot q-1\right)} .
\end{aligned}
$$

Notice that $s=\varrho^{2}+4$. Then $\theta$ denotes the scattering angle. We will return to this later. The collision operator $Q$ satisfies the following important cancelation property:

$$
\int_{\mathbb{R}^{3}} Q(g, g)(p)\left(\begin{array}{c}
1 \\
p \\
p^{0}
\end{array}\right) d p=0,
$$

which leads to the conservation laws of mass, momentum, and energy:

$$
\frac{d}{d t} \int_{\mathbb{R}^{3}} g(p, t) d p=0, \quad \frac{d}{d t} \int_{\mathbb{R}^{3}} g(p, t) p d p=0, \quad \frac{d}{d t} \int_{\mathbb{R}^{3}} g(p, t) p^{0} d p=0 .
$$

Smooth solutions satisfy the following celebrated entropy dissipation:

$$
\frac{d}{d t} \int_{\mathbb{R}^{3}} g \log g d p \leq 0
$$

Steady states of the relativistic Boltzmann equation are the relativistic Maxwellians, which are also known as the Jüttner solutions, which, after normalization, take the following form:

$$
J(p)=\frac{e^{-p^{0}}}{4 \pi} .
$$

The mathematical study of the relativistic Boltzmann equation has a relatively short history. In the near-global-Maxwellian regime, Dudyński and Ekiel-Jeżewska studied the linearized relativistic Boltzmann equation in $[24,25]$ using the spectral analysis developed by Ukai [54]. The nonlinear relativistic Boltzmann equation in a periodic domain was then considered by Glassey and Strauss [31]. This was extended to the whole space in [32] by the same authors. In this paper, they derived the center of mass representation for the relativistic collision operator. Guo and Strain extended 
and developed the energy method originally due to Guo $[34,35]$ to study the relativistic Vlasov-Maxwell-Landau equation [37] and the relativistic Vlasov-MaxwellBoltzmann equation [36]. In the latter case, the two equivalent representations for the collision integral - namely, the center of mass framework and the center of momentum framework - are used interchangeably to avoid the singularity arising in the relativistic collision operator. A systematic derivation of these two representations for the collision operator can be found in [51]. Glassey proved the existence of mild solutions when the initial data lies in the near-vacuum regime [29], as did Strain [52] under different assumptions. It is proved in [5] that the relativistic Boltzmann equation without loss term can blow up. For the Newtonian limit of the relativistic Boltzmann equation, see $[12,52]$. We refer to $[22,23,40]$ for the study of the relativistic Boltzmann equation for general large solutions in the framework of the renormalized solution developed in $[20,21]$. (See also $[42,43,44]$.$) Andréasson [4] proved that the gain term Q^{+}$of the relativistic collision operator enjoys the similar gain of regularity as in the classical case. For general mathematical and physical reference on the relativistic or Newtonian Boltzmann equation, see [15, 16, 17, 18, 27, 30, 56].

In this paper, we are interested in the case where the dynamics of the gas does not depend on the spatial variable, that is, $g(x, p, t)=g(p, t)$. In this case, the Cauchy problem for (1.1) reads

$$
\partial_{t} g=Q(g, g), \quad g(p, 0)=g_{0}(p), \quad p \in \mathbb{R}^{3} .
$$

The spatially homogeneous Boltzmann equation describes a particle system where the dynamics is stationary in the macroscopic scale, and therefore, no significant macroscopic behavior is observed. This, however, does not necessarily mean that the gas is in the equilibrium state, because the dynamics at the microscopic level is still very active and complicated, which is represented by the collision operator. It can also be considered as a model equation for the full Boltzmann equation to gain some experience and intuition about the equation. For these reasons, the spatially homogeneous Boltzmann equation for classical particles has been extensively studied. Early mathematically rigorous studies can be traced back to Carleman [13]. For the Cauchy problem see $[7,8,14,45,46,55]$. Convergence to the thermodynamic equilibrium was studied in $[1,9,47]$. Various $L^{p}$ estimates and their applications were considered in $[6,38,39,48]$. For the propagation and creation of various types of moments estimates, see $[2,3,7,8,10,11,19,26,28,49,53,45]$. It seems that the spatially homogeneous Boltzmann equation is very well understood by now, but very few works have studied the relativistic case. In [41], the Cauchy problem for the spatially homogeneous Boltzmann equation is studied in the context of cosmology. Our emphasis is more on the qualitative properties such as various moment estimates and the relativistic properties of the collision frequency.

One of the reasons the homogeneous theory for the Boltzmann equation has been so successful is because of the propagation and even finite time appearance of $L^{1}$ velocity moments. This is not the case for the general inhomogeneous Boltzmann equation. Such moment estimates play key roles in virtually every aspect of the homogeneous theory.

The starting point for such moment estimates is the so-called Povzner inequality [49]. It has various versions by many authors, but it can be written roughly as

$$
\left|v^{\prime}\right|^{k}+\left|v_{*}^{\prime}\right|^{k}-|v|^{k}-\left|v_{*}\right|^{k} \leq-C_{k}\left\{|v|^{k}+\left|v_{*}\right|^{k}\right\}+\text { (lower order terms), }
$$

where $\left(v, v_{*}\right)$ and $\left(v^{\prime}, v_{*}^{\prime}\right)$ denote the precollisional and postcollisional velocity pairs for the Newtonian particles, respectively. The cases $k=0$ and $k=2$ correspond 
to the conservation laws of mass and energy. Therefore, the Povzner inequality can be considered as an extension of the conservation laws to general exponents larger than 2. Basically, it says that although we cannot expect exact cancellations for general $k>2$, we can still extract a good term which enables us to derive various nice moment estimates $[3,7,8,10,11,19,26,28,53,45]$. In this paper, we start from establishing the relativistic version of the Povzner inequality below $\mathbb{S}_{+}^{2}=\left\{\omega \in \mathbb{S}^{2}\right.$ : $\left.\omega \cdot\left(p / p^{0}-q / q^{0}\right) \geq 0\right\}$ denotes the upper hemisphere in $\mathbb{R}^{3}$,

$$
\begin{aligned}
& \int_{\mathbb{S}_{+}^{2}} \sigma(\varrho, \theta)\left\{\left(p^{\prime 0}\right)^{k}+\left(q^{\prime 0}\right)^{k}-\left(p^{0}\right)^{k}-\left(q^{0}\right)^{k}\right\} d \omega \\
& \quad \leq-\left(1-C_{p} a_{k}\right)\left\{\left(p^{0}\right)^{k}+\left(q^{0}\right)^{k}\right\}+C_{p} a_{k}\left\{\left(p^{0}+q^{0}\right)^{k}-\left(p^{0}\right)^{k}-\left(q^{0}\right)^{k}\right\}
\end{aligned}
$$

for some constants $a_{k}$ and $C_{p}$. The key point is the use of the exponent of microscopic energy $p^{0}$, instead of the modulus of momentum $|p|$ as the polynomial weight for the moments. More precisely, we consider the formula for the microscopic energy for postcollisional particles in the center of momentum coordinates (see section 2). Such a choice - using the center of momentum coordinates instead of the center of mass coordinates and using the microscopic energy as a key object instead of the size of momentum - considerably simplifies calculations and enables us to follow the standard argument developed for the Newtonian case. After the derivation of the Povzner inequality, we aim at deriving a differential inequality for the velocity moments. Then, to utilize the good negative term in the Povzner inequality, we need to estimate the loss part of the collision operator from below uniformly in time. This is considered in Lemma 3.3 (see (2.1) in section 2 for the definition of $v_{\varnothing}$ ):

$$
\int v_{\varnothing} \sigma(\varrho, \theta) g(q, t) d \omega d q \geq C_{f_{0}}\left(p^{0}\right)^{b} .
$$

We point out that several ideas used in the proof of the analogous inequality in the classical case fail under special relativity. This is mainly because there is a fundamental difference between the relativistic collision kernel and the classical one. First, the lower bound for the Møller velocity $v_{\varnothing}$ involves a nontrivial denominator which complicates the estimate. On the other hand, the lack of some uniformity of the collision kernel causes additional difficulties. The collision kernel for the Newtonian Boltzmann equation is uniformly estimated using the Galilean invariance as $\int_{B\left(v-v_{*}, \omega\right) \leq R} d v_{*}=\int_{B\left(v_{*}\right) \leq R} d v_{*}=C R^{3}$ in the hard sphere case. (See [17].) This considerably simplifies the proof in the Newtonian case. It is uniform in the sense that the estimate is independent of $v$.

We do not, of course, have Galilean invariance for the relativistic case. But if we can control the size of the set $\left\{q \mid v_{\varnothing}(p, q) \leq A\right\}$, we still can follow the same line of proof as in the Newtonian case. More precisely, it is desirable to show that the Lebesgue measure of this set is as small as we want uniformly with respect to $p$ by taking $A$ sufficiently small. In Lemma 3.4, we prove, however, that such uniformity does not exist for the relativistic Boltzmann equation in the sense that no matter how small $A$ is, there are four-momentum $p^{\mu}$ such that

$$
h_{p^{\mu}}(A)=m\left(\left\{q^{\mu} \mid v_{\varnothing}(p, q) \leq A\right\}\right)=\infty,
$$

where $m(B)$ denotes the Lebesgue measure of the measurable set $B$. Therefore, the size of the set of momentum which makes the kinetic part of the collision kernel small 
can be arbitrarily large, which is in sharp constrast with the Newtonian Boltzmann equation with the hard potential interaction. This shows that there is a fundamental difference between the classical Boltzmann equation and the relativistic case, and dynamics for the latter are much more complicated.

Throughout this paper, we assume that the differential cross section $\sigma$ satisfies the following properties:

- Hypothesis on the differential cross section: Assumption $(\mathcal{H})$ :

1. $\sigma$ is decomposed into the kinetic part and the angular part:

$$
\sigma(\varrho, \theta)=\varrho^{b} \sigma_{0}(\theta) \quad(0 \leq b \leq 1) .
$$

2. $\sigma_{0}(\theta)$ is nonnegative and bounded in $L^{p}$ for $p>1$ :

$$
\sigma_{0}(\theta) \geq 0, \quad\left\|\sigma_{0}\right\|_{L^{p}\left(\mathbb{S}^{2}\right)}^{p} \equiv \int_{\mathbb{S}^{2}}\left|\sigma_{0}(\theta)\right|^{p} d \omega<\infty .
$$

Note that since $\mathbb{S}^{2}$ is compact, $\sigma_{0} \in L^{p}\left(\mathbb{S}^{2}\right)$ implies $\sigma_{0} \in L^{1}\left(\mathbb{S}^{2}\right)$. From now on, for computational simplicity, we normalize the $L^{1}$ norm of $\sigma_{0}$ without loss of generality as

$$
\int_{\mathbb{S}^{2}} \sigma_{0}(\theta) d \omega=1
$$

Notation. We set some notation used throughout this paper.

- We employ the following notations for a weighted $L^{1}$ norm:

$$
\|g\|_{L_{k}^{1}} \equiv \int_{\mathbb{R}^{3}}|g(p)|\left(p^{0}\right)^{k} d p .
$$

- For the case $k=0$, we use the simplified notation when there's no risk of confusion:

$$
\|g\|_{L^{1}} \equiv \int_{\mathbb{R}^{3}}|g(p)| d p .
$$

This paper is organized as follows. In section 2, we represent the two equivalent reductions of the collision integral, namely, the center of mass representation and the center of momentum representation. In section 3, we derive the relativistic version of the Povzner inequality and the lower bound of the collision frequency on the loss term. A counterexample is given which shows that the Møller velocity cannot be uniformly controlled. In section 4, the Cauchy problem is studied and we consider existence and uniqueness. Section 5 is devoted to the propagation of moments in $L^{1}$.

2. Reduction of the collision operator. In this section, we present two equivalent representations of the collision integral, which was derived by performing the Dirac delta reduction from (1.2). Although we use only the center of momentum framework, we present both in detail for completeness. For the details of the derivation, see [50].

2.1. Glassey-Strauss reduction of the collision operator. In this framework, $Q$ takes the following explicit form:

$$
Q(F, G)=\int_{\mathbb{R}^{3} \times \mathbb{S}_{+}^{2}} \frac{s \sigma(\varrho, \theta)}{p^{0} q^{0}} B(p, q, \omega)\left\{F\left(p^{\prime}\right) G\left(q^{\prime}\right)-F(p) G(q)\right\} d \omega d q
$$


The kernel $B$ is given by

$$
B(p, q, \omega)=\frac{\left(p^{0}+q^{0}\right)^{2} p^{0} q^{0}\left|\omega \cdot\left(\frac{p}{p^{0}}-\frac{q}{q^{0}}\right)\right|}{\left[\left(p^{0}+q^{0}\right)^{2}-(\omega \cdot[p+q])^{2}\right]^{2}} .
$$

Here $(p, q)$ and $\left(p^{\prime}, q^{\prime}\right)$ denote the momentum pairs before and after the collision, respectively. The microscopic conservation laws give the following explicit relation:

$$
p^{\prime}=p-a(p, q, \omega) \omega, \quad q^{\prime}=q+a(p, q, \omega) \omega, \quad \omega \in \mathbb{S}_{+}^{2},
$$

where $a$ is given by

$$
a(p, q, \omega)=\frac{2\left(p^{0}+q^{0}\right) p^{0} q^{0}\left|\omega \cdot\left(\frac{p}{p^{0}}-\frac{q}{q^{0}}\right)\right|}{\left(p^{0}+q^{0}\right)^{2}-(\omega \cdot[p+q])^{2}} .
$$

The expression for the energy takes the following form:

$$
p^{\prime 0}=p^{0}+N_{0}(p, q, \omega), \quad q^{\prime 0}=q^{0}+N_{0}(p, q, \omega),
$$

where $N_{0}$ stands for

$$
N_{0}=\frac{2 \omega \cdot(p+q)\left\{p^{0}(\omega \cdot q)-q^{0}(\omega \cdot p)\right\}}{\left(p^{0}+q^{0}\right)^{2}-\{\omega \cdot(p+q)\}^{2}} .
$$

The Jacobian for the change of variable $\left(p^{\prime}, q^{\prime}\right) \rightarrow(p, q)$ can be calculated as (see [33])

$$
\frac{\partial\left(p^{\prime}, q^{\prime}\right)}{\partial(p, q)}=-\frac{p^{\prime 0} q^{\prime 0}}{p^{0} q^{0}}
$$

Finally, the scattering angle $\theta$ in this case is given by

$$
\cos \theta=\frac{\left(p^{\mu}-q^{\mu}\right)\left(p_{\mu}^{\prime}-q_{\mu}^{\prime}\right)}{\varrho^{2}}=\frac{-\left(p^{0}-q^{0}\right)\left(p^{0}-q^{0}\right)+(p-q) \cdot\left(p^{\prime}-q^{\prime}\right)}{\varrho^{2}} .
$$

These calculations can be found in [51].

2.2. Center of momentum reduction of the collision operator. Another reduction was carried out in $[18,51]$, where the collision operator can be written as

$$
Q(f, g)=\int_{\mathbb{R}^{3} \times \mathbb{S}_{+}^{2}} v_{\varnothing} \sigma(\varrho, \theta)\left\{f\left(p^{\prime}\right) g\left(q^{\prime}\right)-g(p) g(q)\right\} d \omega d q .
$$

The Møller velocity $v_{\varnothing}$ is defined by

$$
v_{\varnothing}=v_{\varnothing}(p, q)=\frac{1}{2} \frac{\varrho \sqrt{s}}{p^{0} q^{0}} .
$$

The relation between the precollisional momentum pair $(p, q)$ and the postcollisional momentum pair $\left(p^{\prime}, q^{\prime}\right)$ in this case is given by

$$
\begin{aligned}
& p^{\prime}=\frac{p+q}{2}+\frac{\varrho}{2}\left(\omega+(\gamma-1)(p+q) \frac{(p+q) \cdot \omega}{|p+q|^{2}}\right), \\
& q^{\prime}=\frac{p+q}{2}-\frac{\varrho}{2}\left(\omega+(\gamma-1)(p+q) \frac{(p+q) \cdot \omega}{|p+q|^{2}}\right),
\end{aligned}
$$


where $\gamma=\left(p^{0}+q^{0}\right) / \sqrt{s}$. The energy for the postcollisional pair satisfies

$$
\begin{aligned}
& p^{\prime 0}=\frac{p^{0}+q^{0}}{2}+\frac{\varrho}{2 \sqrt{s}} \omega \cdot(p+q), \\
& q^{\prime 0}=\frac{p^{0}+q^{0}}{2}-\frac{\varrho}{2 \sqrt{s}} \omega \cdot(p+q) .
\end{aligned}
$$

The angle $\theta$ in the differential cross section $\sigma$ is defined by

$$
\cos \theta=\frac{k}{|k|} \cdot \omega
$$

where the vector $k$ denotes

$$
k=-\frac{p+q}{\sqrt{s}}\left(p^{0}+q^{0}\right)+(p-q)+(\varrho-1)(p+q) \frac{(p+q) \cdot(p-q)}{|p+q|^{2}} .
$$

These calculations can be found in [51].

3. Relativistic Povzner inequality. First, we record the following useful technical lemma from [31].

Lemma 3.1 (see [31]). @ satisfies the following lower and upper bounds:

$$
\frac{|p-q|^{2}+|p \times q|^{2}}{2 p^{0} q^{0}} \leq \varrho^{2} \leq \frac{1}{2}|p-q|^{2} .
$$

In the following lemma, we derive the relativistic version of the Povzner inequality.

Lemma 3.2. Suppose $\sigma(\rho, \theta)$ satisfies the hypothesis $(\mathcal{H})$. Then the relativistic momentum satisfy the following Povzner-type inequality:

$$
\begin{aligned}
& \int_{\mathbb{S}_{+}^{2}} \sigma(\varrho, \theta)\left\{\left(p^{\prime 0}\right)^{k}+\left(q^{\prime 0}\right)^{k}-\left(p^{0}\right)^{k}-\left(q^{0}\right)^{k}\right\} d \omega \\
& \quad \leq-\left(1-C_{p} a_{k}\right) \varrho^{b}\left\{\left(p^{0}\right)^{k}+\left(q^{0}\right)^{k}\right\}+C_{p} a_{k} \varrho^{b}\left\{\left(p^{0}+q^{0}\right)^{k}-\left(p^{0}\right)^{k}-\left(q^{0}\right)^{k}\right\},
\end{aligned}
$$

where $C_{p}=2\left\|\sigma_{0}\right\|_{L^{p}\left(\mathbb{S}^{2}\right)}, a_{k}=1 /(1+k q)^{\frac{1}{q}},(1 / p+1 / q=1)$ and we suppose that $k>1$.

Proof. We observe from the center of momentum representation that

$$
\begin{aligned}
p^{\prime 0} & =\left|\frac{p^{0}+q^{0}}{2}+\frac{\varrho}{2 \sqrt{k}}(p+q) \cdot \omega\right| \\
& =\left(\frac{p^{0}+q^{0}}{2}\right)\left|1+\frac{\varrho}{\sqrt{k}} \frac{|p+q|}{p^{0}+q^{0}}\left(\frac{(p+q)}{|p+q|} \cdot \omega\right)\right| .
\end{aligned}
$$

Similarly $q^{\prime 0}$ can be written as

$$
q^{\prime 0}=\left(\frac{p^{0}+q^{0}}{2}\right)\left|1-\frac{\varrho}{\sqrt{s}} \frac{|p+q|}{p^{0}+q^{0}}\left(\frac{(p+q)}{|p+q|} \cdot \omega\right)\right| .
$$


Therefore, if we set

$$
A(p, q)=\frac{\varrho}{\sqrt{s}} \frac{|p+q|}{p^{0}+q^{0}}, \quad B(p, q)=\frac{p+q}{|p+q|} \cdot \omega
$$

then we have

$$
\begin{aligned}
& \int_{\mathbb{S}_{+}^{2}} \sigma(\varrho, \theta)\left\{\left(p^{\prime 0}\right)^{k}+\left(q^{\prime 0}\right)^{k}\right\} d \omega \\
& \quad=\int_{\mathbb{S}_{+}^{2}}\left(\frac{p^{0}+q^{0}}{2}\right)^{k} \sigma(\varrho, \theta)\left\{|1+A(p, q) B(p, q)|^{k}+|1-A(p, q) B(p, q)|^{k}\right\} d \omega .
\end{aligned}
$$

We then observe that

$$
\left(p^{0}+q^{0}\right)^{2}-|p+q|^{2}=2+2 \sqrt{1+|p|^{2}} \sqrt{1+|q|^{2}}-2 p \cdot q \geq 0,
$$

and $s=\varrho^{2}+4 \geq 0$, which implies

$$
|A(p, q)|=\frac{\varrho}{\sqrt{s}} \frac{|p+q|}{p^{0}+q^{0}} \leq 1 .
$$

Now, it can be easily verified that the function $h(t)$ defined by

$$
h(t)=|1+t|^{k}+|1-t|^{k} \quad(0 \leq t \leq 1)
$$

is a nondecreasing function of $t$. As a consequence, we have from (3.1) that

$$
\begin{aligned}
\int_{\mathbb{S}_{+}^{2}} \sigma(\varrho, \theta)\left\{\left(p^{0}\right)^{k}+\left(q^{\prime 0}\right)^{k}\right\} d \omega & =\left(\frac{p^{0}+q^{0}}{2}\right)^{k} \int_{\mathbb{S}_{+}^{2}} \sigma(\varrho, \theta) h(A(p, q) B(p, q)) d \omega \\
& \leq\left(\frac{p^{0}+q^{0}}{2}\right)^{k} \int_{\mathbb{S}_{+}^{2}} \sigma(\varrho, \theta) h(B(p, q)) d \omega \\
& =\left(p^{0}+q^{0}\right)^{k} G(p, q),
\end{aligned}
$$

where $G$ denotes

$$
G(p, q)=\frac{1}{2^{k}} \int_{\mathbb{S}_{+}^{2}} \sigma(\varrho, \theta)\left\{|1+B(p, q)|^{k}+|1-B(p, q)|^{k}\right\} d \omega .
$$

From our assumption $(\mathcal{H})$ on $\sigma$, and a classical calculation, $G(p, q)$ can be estimated as

$$
\begin{aligned}
G(p, q) & \leq \varrho^{b} \int_{\mathbb{S}_{+}^{2}} \frac{1}{2^{k}} \sigma_{0}(\theta)\left\{\left|\left(1-\frac{p+q}{|p+q|} \cdot \omega\right)\right|^{k}+\left|\left(1+\frac{p+q}{|p+q|} \cdot \omega\right)\right|^{k}\right\} d \omega \\
& \leq 2 \varrho^{b}\left\|\sigma_{0}\right\|_{L^{p}\left(\mathbb{S}^{2}\right)}\left\{\int_{\mathbb{S}^{2}}\left|\frac{1}{2}\left(1+\frac{p+q}{|p+q|} \cdot \omega\right)\right|^{q k} d \omega\right\}^{\frac{1}{q}} \\
& \leq C_{p} \varrho^{b}\left\{\int_{-1}^{1}\left(\frac{1+\mu}{2}\right)^{k q} d \mu\right\}^{\frac{1}{q}} \\
& \leq C_{p} a_{k} \varrho^{b},
\end{aligned}
$$


where $C_{p}$ and $a_{k}$ are given by $C_{p}=2\left\|\sigma_{0}\right\|_{L^{p}\left(\mathbb{S}^{2}\right)}$ and $a_{k}=1 /(1+k q)^{\frac{1}{q}}$, respectively. This yields

$$
\int_{\mathbb{S}_{+}^{2}} \sigma(\varrho, \theta)\left\{\left(p^{\prime 0}\right)^{k}+\left(q^{\prime 0}\right)^{k}\right\} d \omega \leq C_{p} a_{k} \varrho^{b}\left\{p^{0}+q^{0}\right\}^{k}
$$

Therefore, recalling the assumptions $(\mathcal{H})$ on the cross section, we have

$$
\begin{aligned}
& \int_{\mathbb{S}_{+}^{2}} \sigma(\varrho, \theta)\left\{\left(p^{\prime 0}\right)^{k}+\left(q^{\prime 0}\right)^{k}-\left(p^{0}\right)^{k}-\left(q^{0}\right)^{k}\right\} d \omega \\
& \quad \leq \varrho^{b}\left\{C a_{k}\left(p^{0}+q^{0}\right)^{k}-\left(p^{0}\right)^{k}-\left(q^{0}\right)^{k}\right\} \\
& \quad \leq \varrho^{b}\left[-\left(1-C a_{k}\right)\left\{\left(p^{0}\right)^{k}+\left(q^{0}\right)^{k}\right\}+C a_{k}\left\{\left(p^{0}+q^{0}\right)^{k}-\left(p^{0}\right)^{k}-\left(q^{0}\right)^{k}\right\}\right] .
\end{aligned}
$$

This completes the proof.

Lemma 3.3. Let $g$ have finite mass, energy, and entropy. Then there exists positive constants $C_{1}$ and $C_{2}$, which are determined only by the mass, energy, and entropy of the initial data $g_{0}$, such that the following estimate holds:

$$
C_{1}\left(p^{0}\right)^{b} \leq \int_{\mathbb{R}^{3} \times \mathbb{S}^{2}} v_{\varnothing} \sigma(\varrho, \theta) g(q) d \omega d q \leq C_{2}\left(p^{0}\right)^{b} .
$$

Under the same conditions we also have that $C_{1}\left(p^{0}\right)^{b} \leq \int_{\mathbb{R}^{3}} v_{\varnothing} \varrho^{b} g(q) d \omega d q \leq C_{2}\left(p^{0}\right)^{b}$.

Proof. The upper bound follows easily from $(\mathcal{H})$ and Lemma 3.1 as

$$
\begin{aligned}
\int_{\mathbb{R}^{3} \times \mathbb{S}^{2}} v_{\varnothing} \sigma(\varrho, \theta) g(q) d \omega d q & \leq C \int_{\mathbb{R}^{3} \times \mathbb{S}^{2}}|p-q|^{b} g(q) d \omega d q \\
& \leq C_{b}|p|^{b} \int g(q) d q+\int|q|^{b} g(q) d q \\
& \leq C_{b}|p|^{b} \int g(q) d q+\int q^{0} g(q) d q \\
& \leq C_{b}\left(|p|^{b}+1\right) \\
& \leq C_{b}\left(p^{0}\right)^{b} .
\end{aligned}
$$

The lower bound is more involved. We first employ Lemma 3.1 to get the following lower bound estimate for the Møller velocity:

$$
v_{\varnothing}=\frac{\varrho \sqrt{1+\varrho^{2}}}{p^{0} q^{0}} \geq \frac{\varrho^{2}}{p^{0} q^{0}} \geq \frac{|p-q|^{2}}{\left(p^{0} q^{0}\right)^{2}} .
$$

With this and the assumption $(\mathcal{H})$, we have

$$
\int_{\mathbb{R}^{3}} v_{\varnothing} \sigma(\varrho, \theta) g(q) d \omega d q \geq \int_{\mathbb{R}^{3}} \frac{|p-q|^{2+b}}{\left(p^{0} q^{0}\right)^{2}} g(q) d q .
$$

We then divide the estimate into the following two cases: 
(i) $|p|<r_{p}$. We estimate the collision frequency from below as follows:

$$
\begin{aligned}
\int_{\mathbb{R}^{3}} \frac{|p-q|^{2+b}}{\left(p^{0} q^{0}\right)^{2}} g(q) d q & \geq 1_{|p|<r_{p}} \int_{\mathbb{R}^{3}} \frac{|p-q|^{2+b}}{\left(p^{0} q^{0}\right)^{2}} g(q) d q \\
& \geq 1_{|p|<r_{p}} \int_{|q|<r_{q}} \frac{|p-q|^{2+b}}{\left(p^{0} q^{0}\right)^{2}} g(q) d q \\
& \geq \frac{1}{\left(1+r_{p}^{2}\right)\left(1+r_{q}^{2}\right)} \int_{|q|<r_{q}}|p-q|^{2+b} g(q) d q \\
& \geq \frac{r_{|p-q|}^{2+b}}{\left(1+r_{p}^{2}\right)\left(1+r_{q}^{2}\right)} \int_{|p-q| \geq r_{|p-q|}}^{\mid r_{\mid}} g(q) d q \\
& \geq \frac{r_{|p-q|}^{2+b}}{\left(1+r_{p}^{2}\right)\left(1+r_{q}^{2}\right)}\left\{1-\int_{|q| \geq r_{q}} g(q) d q-\int_{|p-q|<r_{|p-q|}} g(q) d q\right\} .
\end{aligned}
$$

We can control the first integral using the conservation of energy:

$$
\begin{aligned}
\int_{|q| \geq r_{q}} g(q) d q & \leq \frac{1}{\left(1+r_{q}^{2}\right)^{\frac{1}{2}}} \int_{\mathbb{R}^{3}} \sqrt{1+|q|^{2}} g(q) d q \\
& =\frac{1}{\left(1+r_{q}^{2}\right)^{\frac{1}{2}}} E\left(g_{0}\right),
\end{aligned}
$$

where $E(h)=\int_{\mathbb{R}^{3}} \sqrt{1+|q|^{2}} h(q) d q$. The second integral is slightly more involved. We first divide the estimate as

$$
\int_{|p-q|<r|p-q|} g(q) d q \leq \int_{\substack{g>B \\|p-q|<r|p-q|}} g(q) d q+\int_{\substack{g<B \\|p-q|<r|p-q|}} g(q) d q .
$$

The second integral is $C r_{|p-q|}^{3} B$ for some constant $C$. We then assume without loss of generality that $B>1$ and we estimate the first integral as

$$
\begin{aligned}
\int_{\substack{g>B \\
|p-q|<r_{|p-q|}}} g(q) d q & \leq \frac{1}{\ln B} \int_{g>B} g \ln g d q \\
& \leq \frac{1}{\ln B} \int_{g>1} g \ln g d q \\
& =\frac{1}{\ln B} \int_{\mathbb{R}^{3}} g \ln g d q-\frac{1}{\ln B} \int_{g<1} g \ln g d q .
\end{aligned}
$$

The first integral is controlled by the finite entropy of $g_{0}$. To estimate the second term, we split the integral as

$$
\begin{aligned}
\int_{g<1} g \ln g d q & \leq \int_{e^{-q^{0}}<g<1} g \ln g d q-\int_{g<e^{-q^{0}}} g \ln g d q \\
& \leq \frac{1}{\ln B}\left\{\int g(q) q^{0} d q+\int_{\mathbb{R}^{3}} e^{-\frac{q^{0}}{2}} d q\right\} \\
& \leq \frac{1}{\ln B}\left\{E\left(g_{0}\right)+C\right\} .
\end{aligned}
$$


In the last line, we used the conservation of energy and the elementary inequality $x \log x \leq C \sqrt{x}(0<x \leq 1)$. This gives

$$
\int_{\substack{g>B \\|p-q|<r_{|p-q|}}} g(q) d q \leq \frac{1}{\ln B}\left\{H\left(g_{0}\right)+E\left(g_{0}\right)+C\right\},
$$

where $H(g)=\int_{\mathbb{R}^{3}} g \log g d p$. We combine the above estimates to obtain for $|p|<r_{p}$

$$
\begin{aligned}
& \int_{\mathbb{R}^{3} \times \mathbb{S}_{+}^{2}} v_{\varnothing} \sigma(\varrho, \theta) g(q) d \omega d q \\
& \quad \geq \frac{r_{|p-q|}^{2+b}}{\left(1+r_{p}^{2}\right)\left(1+r_{q}^{2}\right)}\left\{1-\frac{E\left(g_{0}\right)}{\sqrt{1+r_{q}^{2}}}-\frac{H\left(g_{0}\right)}{\ln B}-\frac{E\left(g_{0}\right)+C}{\ln B}-C r_{|p-q|}^{3} B\right\} .
\end{aligned}
$$

(ii) $|p| \geq r_{p}$. We first note that $\sqrt{1+|p|^{2}}$ is Lipschitz continuous:

$$
\left|p^{0}-q^{0}\right| \leq C|p-q|
$$

for some positive constant $C$. We then employ an elementary inequality,

$$
\frac{x^{2+b}}{2^{1+b}}-y^{2+b} \leq|x-y|^{2+b}, \quad x, y \geq 0, \quad b \geq 0,
$$

to find

$$
|p-q|^{2+b} \geq C\left\{\frac{\left(p^{0}\right)^{2+b}}{2^{1+b}}-\left(q^{0}\right)^{2+b}\right\}
$$

for some constant $C$. Hence we have

$$
\begin{aligned}
\int_{\mathbb{R}^{3}} \frac{|p-q|^{2+b}}{\left(p^{0} q^{0}\right)^{2}} g(q) d q & \geq C \int_{\mathbb{R}^{3}} \frac{2^{-(1+b)}\left(p^{0}\right)^{2+b}-C\left(q^{0}\right)^{2+b}}{\left(p^{0} q^{0}\right)^{2}} g(q) d q \\
& =C_{b}\left(p^{0}\right)^{b} \int_{\mathbb{R}^{3}} \frac{1}{\left(q^{0}\right)^{2}} g(q) d q-\frac{C}{\left(p^{0}\right)^{2}} \int_{\mathbb{R}^{3}}\left(q^{0}\right)^{b} g(q) d q \\
& =\mathrm{I}+\mathrm{II},
\end{aligned}
$$

where $C_{b}=2^{-(1+b)} C$. We first estimate I from below as follows:

$$
\begin{aligned}
\mathrm{I} & \geq C_{b}\left(p^{0}\right)^{b} \int_{|q| \leq r_{q}} \frac{1}{\left(q^{0}\right)^{2}} g(q) d q \\
& \geq \frac{C_{b}\left(p^{0}\right)^{b}}{\left(1+r_{q}^{2}\right)} \int_{|q| \leq r_{q}} g(q) d q \\
& \geq \frac{C_{b}\left(p^{0}\right)^{b}}{\left(1+r_{q}^{2}\right)}\left(1-\int_{|q| \geq r_{q}} g(q) d q\right) \\
& \geq \frac{C_{b}\left(p^{0}\right)^{b}}{\left(1+r_{q}^{2}\right)}\left(1-\frac{1}{\left(1+r_{q}^{2}\right)^{\frac{1}{2}}} E\left(g_{0}\right)\right) .
\end{aligned}
$$


In the last line, we used the conservation of energy. To estimate the second term, we recall that we are restricting to the case $|p| \geq r_{p}$ to see that

$$
\begin{aligned}
\mathrm{II} & \geq-\frac{C}{\left(p^{0}\right)^{2}} \int_{\mathbb{R}^{3}}\left(q^{0}\right)^{b} g(q) d q \\
& \geq-\frac{C}{1+r_{p}^{2}} \int_{\mathbb{R}^{3}}\left(q^{0}\right)^{b} g(q) d q \quad\left(\Leftarrow|p|>r_{p}\right) \\
& \geq-\frac{C E\left(g_{0}\right)}{1+r_{p}^{2}} \\
& \geq-\frac{C\left(p^{0}\right)^{b}}{1+r_{p}^{2}} .
\end{aligned}
$$

In the last line, we assumed without loss of generality that $r_{p}>E\left(g_{0}\right)^{\frac{1}{b}}$ to get

$$
\int_{\mathbb{R}^{3}}\left(q^{0}\right)^{b} g(q) d q \leq E\left(g_{0}\right) \leq\left(r_{p}\right)^{b}<|p|^{b}<\left(p^{0}\right)^{b} .
$$

Therefore, we conclude for sufficiently large $r_{p}$, when $|p| \geq r_{p}$, that

$$
\int_{\mathbb{R}^{3}} v_{\varnothing} g(q) d q \geq\left(p^{0}\right)^{b}\left\{\frac{C_{b}}{2\left(1+r_{q}^{2}\right)}\left(1-\frac{1}{\left(1+r_{q}^{2}\right)^{\frac{1}{2}}} E\left(g_{0}\right)\right)-\frac{C}{1+r_{p}^{2}}\right\} .
$$

From (3.2) and (3.3), we see that by choosing $r_{p}, r_{q}, B$ sufficiently large and taking $r_{|p-q|}$ sufficiently small, we can get the desired lower bound.

Let us stop here for a while to discuss what feature of the relativistic kernel has complicated the situation. Let us define

$$
h_{p^{\mu}}(A)=m\left(\left\{q^{\mu} \mid v_{\varnothing}\left(p^{\mu}, q^{\mu}\right) \leq A\right\}\right),
$$

where $m(S)$ denotes the three-dimensional Lebesque measure in $\mathbb{R}^{3}$ of $S$. If we have uniform control on the size of the Møller velocity, that is, $\lim _{A \rightarrow 0} h_{p}(A)=0$ uniformly with respect to $p$, we would have much simpler proof using

$$
\begin{aligned}
\int_{\mathbb{R}^{3}} v_{\phi} g(q) d q & \geq A\left(1-\int_{v_{\infty} \leq A} g(q) d q\right) \\
& \geq A\left(1-\int_{\left\{v_{\phi} \leq A\right\} \cap\{g \geq B\}} g(q) d q-\int_{\left\{v_{\phi} \leq A\right\} \cap\{g \leq B\}} g(q) d q\right) \\
& \geq A C,
\end{aligned}
$$

where $C$ is a constant determined by $H\left(g_{0}\right), E\left(g_{0}\right), h_{p}(A)$, and $B$. Such a uniform estimate on the collision kernel is trivial for the classical Boltzmann equation since it is a direct consequence of the Galilean invariance of the kinetic collision kernel, which is, of course, not the case in the special relativistic situation. But the lack of Galilean invariance does not necessarily imply the failure of such estimates since these two properties are by no means equivalent. In the following lemma, we provide an explicit counterexample. It shows that the set of momentum on which the scattering kernel is small can be arbitrarily large. Such phenomena do not happen in the Newtonian case due to the Galilean invariance. This shows that there's a fundamental difference between the Newtonian and the relativistic case. 
Lemma 3.4. Fix $A \ll 1$ and let $\epsilon<A-A^{2}+A^{3}$. Take $p^{\mu} \in \mathbb{R}^{4}$ as

$$
p^{\mu}=\left(\sqrt{\epsilon^{-1}}, \sqrt{\epsilon^{-1}-1}, 0,0\right),
$$

and define a set $S$ by

$$
S=\left\{\left.q^{\mu} \in \mathbb{R}^{4}\left|q_{1}>A_{\varepsilon},\right| q_{2}\right|^{2}+\left|q_{3}\right|^{2}<A\left|q_{1}\right|^{2}\right\},
$$

where $A_{\varepsilon}$ denotes

$$
A_{\varepsilon}=\frac{1-A}{\sqrt{1-\epsilon-(1+A)(1-A)^{2}}}
$$

Then for any $q^{\mu} \in S$, we have

$$
h_{p^{\mu}}(A)=\infty
$$

Proof. We first note that

$$
\frac{p}{\sqrt{1+|p|^{2}}}=(\sqrt{1-\epsilon}, 0,0)
$$

which implies

$$
\frac{p}{\sqrt{1+|p|^{2}}} \cdot \frac{q}{\sqrt{1+|q|^{2}}}=\sqrt{1-\epsilon}\left\{\frac{q_{1}}{\sqrt{1+|q|^{2}}}\right\} .
$$

Now, we take any $q^{\mu}$ from $S$. Then with our choice of $p$ and $q$, it can be verified by straightforward calculation that

$$
0<1-\sqrt{1-\epsilon}\left(\frac{q_{1}}{\sqrt{1+|q|^{2}}}\right) \leq 1-\sqrt{1-\epsilon}\left(\frac{q_{1}}{\sqrt{1+(1+A) q_{1}^{2}}}\right) .
$$

We recall the assumption on $q_{1}$ :

$$
q_{1}^{2}>\frac{(1-A)^{2}}{1-\varepsilon-(1+A)(1-A)^{2}} .
$$

We multiply $\left(1-\varepsilon-(1+A)(1-A)^{2}\right)(1-\varepsilon)^{-1}$ on both sides:

$$
\left\{1-(1+A) \frac{(1-A)^{2}}{1-\varepsilon}\right\} q_{1}^{2}>\frac{(1-A)^{2}}{1-\varepsilon} .
$$

We gather relevant terms as

$$
\frac{(1-A)^{2}}{1-\varepsilon}\left\{1+(1+A) q_{1}^{2}\right\}<q_{1}^{2} .
$$

We then divide both sides by $1+(1+A) q_{1}^{2}$ and take the square root on both sides to get

$$
\frac{1-A}{\sqrt{1-\varepsilon}}<\frac{q_{1}}{\sqrt{1+(1+A) q_{1}^{2}}}
$$


Hence we have

$$
1-\sqrt{1-\epsilon}\left(\frac{q_{1}}{\sqrt{1+(1+A) q_{1}^{2}}}\right)<A .
$$

We collect (3.4), (3.5), and (3.6) to find

$$
\begin{aligned}
\left\{1-\frac{p}{\sqrt{1+|p|^{2}}} \cdot \frac{q}{\sqrt{1+|q|^{2}}}\right\}^{2} & \leq\left\{1-\sqrt{1-\epsilon}\left(\frac{q_{1}}{\sqrt{1+(1+A) q_{1}^{2}}}\right)\right\}^{2} \\
& \leq A^{2}<A^{2}+\frac{1}{\left(1+|p|^{2}\right)\left(1+|q|^{2}\right)}
\end{aligned}
$$

which leads to

$$
v_{\varnothing}^{2}(p, q)=\frac{\left(\sqrt{1+|p|^{2}} \sqrt{1+|q|^{2}}-p \cdot q\right)^{2}-1}{\left(1+|p|^{2}\right)\left(1+|q|^{2}\right)} \leq A^{2} .
$$

Therefore, we have

$$
S \subset\left\{q \mid v_{\varnothing}(p, q) \leq A\right\} .
$$

This proves the lemma because $S$ has infinite Lebesgue measure.

4. Cauchy problem for the homogeneous relativistic Boltzmann equation. To be on a firm basis, we first need to establish the existence of solutions, which the authors couldn't locate in the existing literature. This can be carried out following the standard argument developed for the classical homogeneous Boltzmann equation. (See, for example, $[7,8,17]$.) Since most of them can be adapted with little modification, we give only a brief sketch of the proof here. Consider the homogeneous relativistic Boltzmann equation corresponding to the initial data $g_{0}$ :

$$
\begin{aligned}
\frac{d g}{d t} & =Q(g, g), \\
g(0) & =g_{0} .
\end{aligned}
$$

We first truncate the collision kernel and obtain the global existence for the truncated problem. For this, we consider the functional

$$
\Phi(g)=g_{0}+\int_{0}^{t} Q_{M}(g, g) d s,
$$

where $Q_{M}(g, g)$ denotes the collision operator with the collision kernel $v_{\varnothing} \sigma$ replaced by $v_{\varnothing} \sigma 1_{v_{\varnothing} \sigma \leq M}$. Here $1_{v_{\varnothing} \sigma \leq M}$ is the usual indicator function of the set of points where $v_{\varnothing} \sigma \leq M$. Then, due to the boundedness of the truncated kernel, it is straightforward to verify that

$$
\|\Phi(g)\|_{L^{1}}=\left\|g_{0}\right\|_{L^{1}}+C T M\|g\|_{L^{1}}^{2}
$$

and

$$
\|\Phi(g)-\Phi(h)\|_{L^{1}} \leq C T M\left(\|g\|_{L^{1}}+\|h\|_{L^{1}}\right)\|g-h\|_{L^{1}}^{2}
$$


These two estimates are sufficient to yield the local in time existence in the time interval $\left[0, T_{M}\right]$ such that $4 C T_{M}\left\|g_{0}\right\|_{L^{1}}<1$. We denote the unique local solution by $g^{M}$. We note that the size of $T_{M}$ depends only on the $L^{1}$ norm of the initial data and $M$. Therefore, due to the conservation of mass, by a standard argument it is enough to prove the positivity of $g^{M}$ to extend it to the global solution. It can be carried out in exactly the same manner as in the classical case using the following equation for some $\mu>0$ :

$$
\partial_{t} h+\mu h=\Gamma_{\mu}^{M}(h), \quad h(0)=g_{0},
$$

where $\Gamma_{\mu}^{M}(h)$ is defined by

$$
\Gamma_{\mu}^{M}(h)=Q_{M}(h, h)+\mu h .
$$

Notice that the solution $g^{M}$ to (4.1) with $Q$ replaced by $Q_{M}$ satisfies (4.2) for $\left[0, T_{M}\right)$. It is standard to show that (4.2) has a unique solution. Therefore, to extend the existence time for $g^{M}$ over $(0, \infty)$, it is sufficient to show that the solution of $(4.2)$ is positive since $T_{M}$ only depends upon the $L^{1}$ norm of $g^{M}$, which is conserved when $g^{M}$ is positive.

To show this, we consider the following iteration scheme:

$$
h_{n}(t)=e^{-\mu t} g_{0}+\int_{0}^{t} e^{-\mu(t-s)} \Gamma_{\mu}^{M}\left(h_{n-1}\right) d s, \quad h_{0}=0 .
$$

We then observe that $\Gamma_{\mu}$ is a positive monotone operator for sufficiently large $\mu$ in the sense that

$$
\Gamma_{\mu}^{M}(f) \geq \Gamma_{\mu}^{M}(g) \quad \text { for } f \geq g \geq 0
$$

This easily leads to

$$
0 \leq h_{0} \leq h_{1} \leq h_{2} \leq \cdots \quad \text { and } \quad \int h_{n} \leq\left\|g_{0}\right\|_{L^{1}} .
$$

Then the monotone convergence theorem gives the existence of a unique positive solution to the truncated problem. Due to the boundedness of the kernel, it is straightforward to show that $g^{M}$ satisfies the conservation of mass, momentum, and energy. The entropy dissipation can be proved by considering the following truncated equation $[7,8]$ :

$$
\begin{aligned}
\frac{d}{d t} g_{j m i}^{M}+A_{j} g_{m i}^{j} & =\Gamma_{j m i}^{M}, \\
g_{j m i}^{M}(0) & =g_{m i 0},
\end{aligned}
$$

where $A_{j}, \Gamma_{j m i}^{M}$, and $g_{m i 0}$ are defined by

$$
\begin{aligned}
A_{j} & =\left\{\int\left(g_{0}(q)+\frac{1}{j} e^{-q^{0}}\right) d q\right\}, \\
\Gamma_{j m i}^{M} & =\min \left\{\Gamma_{\mu}^{M}\left(g_{j m i}^{M}\right), i\right\}, \\
g_{m i 0} & =\min \left\{g_{0}(p)+\frac{1}{m} e^{-p^{0}}, i\right\} .
\end{aligned}
$$


We first establish a formal entropy dissipation inequality for $g_{j m i}^{M}$ and then carefully carry out the limiting process $i, m, h \rightarrow \infty$. Since this is almost identical to the proof given in $[7,8]$, we omit it. We now summarize the result in the following theorem.

Proposition 4.1. Let T be an arbitrary positive number. Suppose that the initial data is positive and the mass, energy, and entropy are initially finite:

$$
g_{0} \geq 0, \quad \int g_{0}\left(1+p^{0}\right) d p<\infty, \quad \int g_{0} \ln g_{0} d p<\infty .
$$

Then there exists a unique positive solution $g^{M} \in C_{+}^{1}\left([0, T] ; L_{1}^{1}\right)$ for (4.1) with the truncated kernel satisfying the conservation of mass, momentum, and energy

$$
\begin{aligned}
\int g^{M}(p, t) d p & =\int g_{0}(p) d p, \quad \int g^{M}(p, t) p d p=\int g_{0}(p) p d p, \\
\int g^{M}(p, t) p^{0} d p & =\int g_{0} p^{0} d p,
\end{aligned}
$$

and the entropy dissipation inequality

$$
\int g^{M}(t) \ln g^{M}(t) d p+\int_{0}^{t} D_{K}\left(g^{M}\right) d s \leq \int g_{0} \ln g_{0} d p,
$$

where $D_{K}$ is obtained by replacing the Møller velocity with the truncated one.

It remains to remove the truncation by letting $M \rightarrow \infty$ and show that $g=$ $\lim _{M \rightarrow \infty} g^{M}$ satisfies (4.1). First, using the conservation laws and the entropy inequality, we find from the Dunford-Pettis criterion that $\left\{f^{M}(t)\right\}_{M}$ is weakly compact in $L^{1}$ for each $t$ and, therefore, there exists a function $f \in L^{\infty}\left([0, T] ; L_{1}^{1}\right)$ where we recall that $\left.L_{a}^{1}=L^{1}\left(\left(p^{0}\right)^{a} d p\right)\right)$ and a subsequence, which we still denote by $\left\{f^{M}\right\}$ such that $f^{M}$ converges weakly to $f$ in $L^{1}$ for each fixed $t \in[0, T]$. By a standard diagonal argument, we extract a dense subset $\left\{t_{j}\right\}$, on which $f^{M}$ converges weakly to $f$. Then it remains to prove the equicontinuity of $f^{M}$ to conclude that $f$ is the solution for the homogeneous relativistic Boltzmann equation (4.1). We first note that the Povzner inequality in Lemma 3.2 trivially implies that

$$
\int_{\mathbb{S}^{2}}\left\{\left(p^{\prime 0}\right)^{k}+\left(q^{\prime 0}\right)^{k}-\left(p^{0}\right)^{k}-\left(q^{0}\right)^{k}\right\} d \omega \leq C_{k}\left\{\left(p^{0}\right)^{k-1} q^{0}+p^{0}\left(q^{0}\right)^{k-1}\right\} .
$$

We multiply $\left(p^{0}\right)^{1+b}$ to (4.1), integrate on $\mathbb{R}_{p}^{3}$, and apply (4.4) to derive

$$
\left\|g^{M}(t)\right\|_{L_{1+b}^{1}} \leq\left\|g_{0}\right\|_{L_{1+b}^{1}}+C \int_{0}^{t}\left\|g^{M}(s)\right\|_{L_{1+b}^{1}}\left\|g^{M}(s)\right\|_{L_{1}^{1}} d s
$$

where the constant $C$ is independent of $M$. The Gronwall's lemma then leads to

$$
\left\|g^{M}(t)\right\|_{L_{1+b}^{1}} \leq C_{T}\left\|g_{0}\right\|_{L_{1+b}^{1}},
$$

which implies

$$
\left\|g^{M}(t)-g^{M}(s)\right\|_{L_{1}^{1}} \leq\left\|\int_{s}^{t} Q(g, g) d \tau\right\|_{L_{1}^{1}} \leq C_{T} \int_{s}^{t}\left\|g_{0}\right\|_{L_{1+b}^{1}}^{2} d \tau \leq C_{T}(t-s) .
$$

From this, we conclude that $g \in C\left([0, T] ; L_{1}^{1}\right)$ by the standard equicontinuity argument. This completes the existence part. The conservation of mass and momentum 
still holds. The entropy dissipation inequality follows from the convexity of the entropy production functional as in [21].

The conservation laws of mass, momentum, and energy hold due to

$$
\int_{\mathbb{R}^{3}} Q^{ \pm}(g, g)(p) p^{0} d p<C\|g\|_{L_{1+b}^{1}}^{2},
$$

which enables one to apply the Fubini theorem and the usual cancellation property of $Q^{ \pm}$.

To prove the uniqueness, let $f$ and $g$ be two solutions corresponding to initial data $g_{0}$ satisfying (4.5):

$$
\partial_{t} f=Q(f, f), \quad \partial_{t} g=Q(g, g) .
$$

Taking the difference, multiplying $\operatorname{sgn}(f(t)-g(t)) p^{0}$ on both sides, and integrating on $\mathbb{R}^{3}$, we find

$$
\begin{aligned}
\frac{d}{d t}\|f(t)-g(t)\|_{L_{1}^{1}} & =\int \operatorname{sgn}(f(t)-g(t)) Q(f(t)+g(t), f(t)-g(t)) p^{0} d p \\
& \leq C\left(\|f(t)\|_{L_{1+b}^{1}}+\|g(t)\|_{L_{1+b}^{1}}\right)\|f(t)-g(t)\|_{L_{1}^{1}}
\end{aligned}
$$

which gives

$$
\|f(t)-g(t)\|_{L_{1}^{1}} \leq C_{T}\left\|f_{0}-g_{0}\right\|_{L_{1}^{1}}
$$

We have given an outline of the proof of the following theorem.

THEOREM 4.2. Let $T$ be an arbitrary positive number. Suppose that the initial data is positive and the mass, energy, and entropy are initially finite:

$$
g_{0} \geq 0, \quad \int g_{0}\left(1+p^{0}\right) d p<\infty, \quad \int g_{0} \ln g_{0} d p<\infty .
$$

We assume further that $g_{0}$ satisfies

$$
\int g_{0}(p)\left(p^{0}\right)^{1+b} d p<\infty
$$

where $b>0$ is from $(\mathcal{H})$. Then there exists a unique positive solution $g \in C_{+}^{1}\left([0, T] ; L^{1}\right)$ for (4.1) satisfying the conservation of mass, momentum, and energy:

$$
\begin{aligned}
\int g(p, t) d p & =\int g_{0}(p) d p, \quad \int g(p, t) p d p=\int g_{0}(p) p d p, \\
\int g(p, t) p^{0} d p & =\int g_{0} p^{0} d p .
\end{aligned}
$$

The entropy is nonincreasing,

$$
\int g(t) \ln g(t) d p+\int_{0}^{t} D(g) d s \leq \int g_{0} \ln g_{0} d p
$$

where the entropy production rate is defined as

$$
D(g)=\int_{\mathbb{R}^{6}} v_{\phi} \sigma(\varrho, \theta)\left\{g\left(p^{\prime}\right) g\left(q^{\prime}\right)-g(p) g(q)\right\} \log \left(\frac{g\left(p^{\prime}\right) g\left(q^{\prime}\right)}{g(p) g(q)}\right) d \omega d p d q .
$$

The authors expect Theorem 4.2 can be sharpend further. For example, it would be possible to remove the condition (4.5) in Theorem 4.1 by adopting the theory of Mischler and Wennberg [46]. The entropy inequality could also be improved into an equality. These issues should be considered in a future work. 
5. Moment estimates in $\boldsymbol{L}^{\mathbf{1}}$. We first derive the following system of differential inequalities.

Lemma 5.1. Define the $k$ th algebraic moment $m_{k}$ as

$$
m_{k}(t)=\int g(p, t)\left(p^{0}\right)^{k} d p
$$

Then, for $k>1$ large enough, and for some positive constants $C_{1}$ and $C_{2}$ which are independent of $k, m_{k}$ satisfies the following differential inequality:

$$
\begin{aligned}
\frac{d m_{k}(t)}{d t} \leq & -C_{1}\left(1-C a_{k}\right) m_{k}^{1+\frac{b}{k}}(t) \\
& +a_{k} C_{2} \sum_{j=1}^{r_{k}}\left(\begin{array}{c}
k \\
j
\end{array}\right)\left\{m_{j+b}(t) m_{k-j}(t)+m_{j}(t) m_{(k-j)+b}(t)\right\},
\end{aligned}
$$

where $a_{k}=(k+1)^{-1}$ and $r_{k}=\left[\frac{k+1}{2}\right]$ is the integer part of $\frac{k+1}{2}$.

Proof. We multiply $\left(p^{0}\right)^{k}$ to both sides of (4.1), integrate over $\mathbb{R}_{p}^{3}$, and use the symmetry property of $Q$ to get

$$
\begin{aligned}
& \frac{d}{d t} \int_{\mathbb{R}^{3}} g(p, t)\left(p^{0}\right)^{k} d p \\
& \quad=\int_{\mathbb{R}^{6}} g(p, t) g(q, t)\left(\frac{1}{4} \int_{\mathbb{S}_{+}^{2}} v_{\varnothing} \sigma(\varrho, \theta)\left\{\left(p^{\prime 0}\right)^{k}+\left(q^{\prime 0}\right)^{k}-\left(p^{0}\right)^{k}-\left(q^{0}\right)^{k}\right\} d \omega\right) d p d q .
\end{aligned}
$$

We then apply Lemma 3.2 to see that for some $C>0$ we have

$$
\begin{aligned}
\frac{d}{d t} \int_{\mathbb{R}^{3}} g(p, t)\left(p^{0}\right)^{k} d p \leq & -\frac{1}{4}\left(1-C a_{k}\right) \int_{\mathbb{R}^{6}} v_{\varnothing} \varrho^{b} g(p, t) g(q, t)\left(\left(p^{0}\right)^{k}+\left(q^{0}\right)^{k}\right) d p d q \\
& +\frac{1}{4} C a_{k} \int_{\mathbb{R}^{6}} v_{\varnothing} \varrho^{b} g(p, t) g(q, t)\left\{\left(p^{0}+q^{0}\right)^{k}-\left(p^{0}\right)^{k}-\left(q^{0}\right)^{k}\right\} d p d q \\
= & \frac{1}{4}\left\{-\left(1-C a_{k}\right) A_{k}+C a_{k} B_{k}\right\} .
\end{aligned}
$$

We estimate $A_{k}$ and $B_{k}$ separately. We use Lemma 3.3 to estimate $A_{k}$ from below as

$$
\begin{aligned}
A_{k} & \equiv \int_{\mathbb{R}^{6}} v_{\varnothing} \varrho^{b} g(p, t) g(q, t)\left\{\left(p^{0}\right)^{k}+\left(q^{0}\right)^{k}\right\} d p d q \\
& =2 \int_{\mathbb{R}^{6}} v_{\varnothing} \varrho^{b} g(p, t) g(q, t)\left(p^{0}\right)^{k} d p d q \\
& =C \int_{\mathbb{R}^{3}}\left\{\int_{\mathbb{R}^{3}} v_{\varnothing} \varrho^{b} g(q, t) d q\right\} g(p, t)\left(p^{0}\right)^{k} d p \\
& \geq C m_{k+b}(t) .
\end{aligned}
$$

To estimate $B_{k}$, we first recall the binomial expansion:

$$
\begin{aligned}
\left(p^{0}+q^{0}\right)^{k}-\left(p^{0}\right)^{k}-\left(q^{0}\right)^{k} & =\sum_{j=1}^{k-1}\left(\begin{array}{c}
k \\
j
\end{array}\right)\left(p^{0}\right)^{j}\left(q^{0}\right)^{k-j} \\
& \leq 2 \sum_{j=1}^{r_{k}}\left(\begin{array}{c}
k \\
j
\end{array}\right)\left(p^{0}\right)^{j}\left(q^{0}\right)^{k-j} .
\end{aligned}
$$


Note that we have used the symmetry of the binomial expansion. Thus we have

$$
B_{k} \leq 2 \sum_{j=1}^{r_{k}}\left(\begin{array}{l}
k \\
j
\end{array}\right) \int_{\mathbb{R}^{6}} v_{\varnothing} \varrho^{b} g(p, t) g(q, t)\left\{\left(p^{0}\right)^{j}\left(q^{0}\right)^{k-j}\right\} d \omega d p d q .
$$

We then use Lemma 3.1 to estimate $B_{k}$ as

$$
\begin{aligned}
B_{k} & \leq C \sum_{j=1}^{r_{k}}\left(\begin{array}{l}
k \\
j
\end{array}\right) \int_{\mathbb{R}^{6}}|p-q|^{b} g(p, t) g(q, t)\left\{\left(p^{0}\right)^{j}\left(q^{0}\right)^{k-j}\right\} d p d q \\
& \leq C \sum_{j=1}^{r_{k}}\left(\begin{array}{l}
k \\
j
\end{array}\right) \int_{\mathbb{R}^{6}}\left\{\left(p^{0}\right)^{b}+\left(q^{0}\right)^{b}\right\} g(p, t) g(q, t)\left\{\left(p^{0}\right)^{j}\left(q^{0}\right)^{k-j}\right\} d p d q \\
& \leq C \sum_{j=1}^{r_{k}}\left(\begin{array}{l}
k \\
j
\end{array}\right)\left\{m_{j+b}(t) m_{k-j}(t)+m_{j}(t) m_{k-j+b}(t)\right\} .
\end{aligned}
$$

Consequently, we have for some constants $C_{1}$ and $C_{2}$

$$
\begin{aligned}
\frac{d m_{k}(t)}{d t} \leq & -C_{1}\left(1-C a_{k}\right) m_{k+b}(t) \\
& +a_{k} C_{2} \sum_{j=1}^{r_{k}}\left(\begin{array}{c}
k \\
j
\end{array}\right)\left\{m_{j+b}(t) m_{k-j}(t)+m_{j}(t) m_{(k-j)+b}(t)\right\} .
\end{aligned}
$$

Finally, we apply Jensen's inequality with respect to the unit measure $g(q) d q$ to estimate $m_{k+b}$ from below as

$$
m_{k+b}=\int_{\mathbb{R}^{3}} g(q)\left(q^{0}\right)^{k\left(1+\frac{b}{k}\right)} d q \geq\left(\int_{\mathbb{R}^{3}} g(q)\left(q^{0}\right)^{k} d q\right)^{1+\frac{b}{k}}=m_{k}^{1+\frac{b}{k}}(t) .
$$

This completes the proof.

From the differential inequality in Lemma 5.1, the following theorem on the creation and propagation of exponential moments in $L^{1}$ can be obtained by using the same arguments as in $[2,10,11,28]$.

THEOREM 5.2. Let $g_{0}$ satisfy the main assumptions in Thoerem 4.2.

1. Assume $b>0$. Then the exponential moment is created and propagated in the sense that there exist positive constants $t_{*}, r$, and $\gamma$ depending on $b$ and $f_{0}$ such that

$$
\int g(p, t) e^{r\left(p^{0}\right)^{b}} d p<\infty \quad \forall t>t_{*}
$$

2. If $g_{0}$ alternatively satisfies

$$
\int g_{0}(p)\left(p^{0}\right)^{k} d p<\infty \quad(k \geq 1)
$$

then the polynomial moments propagate in the sense that

$$
\int g(p, t)\left(p^{0}\right)^{k} d p<\infty, \quad t \in \mathbb{R}_{+} .
$$


3. If $g_{0}$ alternatively satisfies for some constant $r_{0}>0$ that

$$
\int g_{0}(p) e^{r_{0} p^{0}} d p<\infty
$$

then the exponential moment will propagate in the sense that there exists a constant $r=r\left(g_{0}, r_{0}\right)>0$ such that

$$
\int g(p, t) e^{r p^{0}} d p<\infty, \quad t \in \mathbb{R}_{+} .
$$

Remark 1. Note that the exponent for $p^{0}$ in part (1) of Theorem 5.2 is exactly the growth rate of the kinetic part of the collision kernel. This optimal result was first established for the classical case in [2]

We mention that the creation of moments holds only when $b>0$. In the case $b=0$, we cannot expect the moments higher than the one satisfied by the initial data to be created. To show this, we consider the following mild form:

$$
g(t)=e^{-\int_{0}^{t} L g d s} g_{0}+\int_{0}^{t} e^{-\int_{s}^{t} L g d \tau} Q^{+}(g, g)(s) d s,
$$

where $L$ denotes

$$
L g=\int v_{\varnothing} \sigma(\varrho, \theta) g(q, t) d \omega d q .
$$

We multiply $\left(p^{0}\right)^{k}$ and integrate on $\mathbb{R}^{3}$ to obtain

$$
\int g(t)\left(p^{0}\right)^{k} d p=\int e^{-\int_{0}^{t} L g d s} g_{0}\left(p^{0}\right)^{k} d p+\int_{0}^{t} \int e^{\int_{s}^{t} L g d \tau} Q^{+}(g, g)(s)\left(p^{0}\right)^{k} d p d s .
$$

For $b=0$, we see from Lemma 3.3 that there exists a constant $C$ depending only on the initial data $g_{0}$ such that

$$
L g=\int v_{\varnothing} \sigma(\varrho, \theta) g(q, t) d \omega d q \leq C .
$$

This leads to

$$
\int g(t)\left(p^{0}\right)^{k} d p \geq \int e^{-C t} g_{0}\left(p^{0}\right)^{k} d p+\int_{0}^{t} \int e^{-C(t-s)} Q^{+}(g, g)(s)\left(p^{0}\right)^{k} d p d s .
$$

Then, since the second term in the right-hand side is nonnegative, we see that finiteness of the $k$ th moment for $g$ at time $t$ inevitably implies $\int g_{0}\left(p^{0}\right)^{k} d p<\infty$. Therefore, for hardball case $b=0$, higher moments cannot appear when it is not bounded initially. The same holds for the Maxwellian molecules in the classical Boltzmann equation (see [53]).

Acknowledgment. The authors would like to gratefully thank both of the referees for their careful reading of this paper.

\section{REFERENCES}

[1] F. Abrahamsson, Strong $L^{1}$ convergence to equibrium without entropy conditions for the Boltzmann equation, Comm. Partial Differential Equations, 24 (1999), pp. 1501-1535. 
[2] R. Alonso, J. A. Canizo, R. Gamba, and C. Mounot, A New Approach to the Creation and Propagation of Exponential Moments in the Boltzmann Equation, Preprint, arXiv:1203.2364, 2012.

[3] R. Alonso And I. M. Gamba, Propagation of $L^{1}$ and $L^{\infty}$ Maxwellian weighted bounds for derivatives of solutions to solutions to the homogeneous elastic Boltzmann equation, J. Math. Pures. Appl., 9 (2007), pp. 575-595.

[4] H. AndrÉAsson, Regularity of the gain term and strong $L^{1}$ convergence to equilibrium for the relativistic Boltzmann equation, SIAM J. Math. Anal., 27 (1996), pp. 1386-1405.

[5] H. Andréasson, S. Calogero, and R. Illner, ON blowup for gain-term-only classical and relativistic Boltzmann equations, Math. Methods Appl. Sci., 27 (2004), pp. 2231-2240.

[6] L. ARKeryd, $L^{\infty}$ estimates for the space-homogeneous Boltzmann equation, J. Stat. Phys., 31 (1983), pp. 347-361.

[7] L. Arkeryd, On the Boltzmann equation I: Existence, Arch. Ration. Mech. Anal., 45 (1972), pp. $1-16$.

[8] L. Arkeryd, On the Boltzmann equation part II: The full initial value problem, Arch. Ration. Mech. Anal., 45 (1972), pp. 17-34.

[9] L. ARKERYD, Stability in $L^{1}$ for the spatially homogeneous Boltzmann equation, Arch. Ration. Mech. Anal., 103 (1988), pp. 151-167.

[10] A. V. Bobylev, I. M. Gamba, and V. A. Panferov, Moment inequalities and high-energy tails for Boltzmann equations with inelastic interactions, J. Stat. Phys., 116 (2004), pp. 1651-1682.

[11] A. V. Bobylev, Moment inqualities for the Boltzmann equation and applications to spatially homogeneous problems, J. Stat. Phys., 88 (1997), pp. 1183-1214.

[12] S. Calogero, The Newtonian limit of the relativistic Boltzmann equation, J. Math. Phys., 45 (2004), pp. 4042-4052.

[13] T. Carleman, Problems mathematiques dans la theorie cineqitue des gas, Almquist \& Wiksells, Uppsala, Sweden, 1957.

[14] E. A. Carlen, M. C. Carvalho, and X. Lu, On strong convergence to equilibrium for the Boltzmann equation with soft potentials, J. Stat. Phys., 135 (2009), pp. 681-736.

[15] C. Cercignani, The Boltzmann Equation and Its Application, Springer-Verlag, New York, 1988.

[16] C. Cercignani and G. Kremer, The Relativistic Boltzmann Equation: Theory and Applications, Prog. Math. Phys. 22, Birkhauser-Verlag, Basel, 2002.

[17] C. Cercignani, R. Illner, and M. Pulvirenti, The Mathematical Theory of Dilute Gases, Appl. Math. Sci. 106, Springer-Verlag, New York, 1994.

[18] S. R. de Groot, W. A. van Leeuwen, Ch. G. van Weert, Relativistic Kinetic Theory, North-Holland, Amsterdam, 1980.

[19] L. Desvillettes, Some applications of the method of moments for the homogeneous Boltzmann and Kac equations, Arch. Ration. Mech. Anal., 123 (1993) pp. 387-404.

[20] R. Diperna And P.-L. Lions, On the Cauchy problem for the Boltzmann equation: Global existence and weak stability, Ann. Math., 130 (1989), pp. 321-366.

[21] R. Diperna And P.-L. Lions, Global solutions of Boltzmann's equation and the entropy inequality, Arch. Ration. Mech. Anal., 114 (1991), pp. 47-55.

[22] M. Dudyński And M. L. EkiEl-Jeżewska, Causality of the linearized relativistic Boltzmann equation, Phys. Rev. Lett., 55 (1985), pp. 2831-2834.

[23] M. Dudyński And M. L. Ekiel-Jeżewska, Global existence proof for relativistic Boltzmann equation, J. Stat. Phys., 66 (1992) pp. 991-1001.

[24] M. Dudyński ANd M. L. EkIEL-JeżEWSKA, On the linearized relativistic Boltzmann equation. I. Existence of solutions, Comm. Math. Phys., 115 (1988), pp. 607-629.

[25] M. Dudý́ski, On the linearized relativistic Boltzmann equation. II. Existence of hydrodynamics, J. Stat. Phys., 57 (1989), pp. 199-245.

[26] T. Elmroth, Global boundedness of moments of solutions of the Boltzmann equation for forces of infinite range, Arch. Ration. Mech. Anal., 82 (1983), pp. 1-12.

[27] M. Escobedo, S. Mischler, And M. A. Vallie, Homogeneous Boltzmann Equation in Quantum Relativistic Kinetic Theory, Electron. J. Differ. Equ. Monogr. 4, Southwest Texas State University, San Marcos, TX, 2003.

[28] I. M. Gamba, V. Panferov, and C. Villani, Upper Maxwellian bounds for the spatially homogeneous Boltzmann equation, Arch. Ration. Mech. Anal., 194 (2009) pp. 253-282.

[29] R. Glassey, Global solutions to the Cauchy problem for the relativistic Boltzmann equation with near-vacuum data, Comm. Math. Phys., 264 (2006), pp. 705-724.

[30] R. Glassey, The Cauchy Problems in Kinetic Theory, SIAM, Philadelphia, 1996. 
[31] R. T. Glassey and W. A. Strauss, Asymptotic stability of the relativistic Maxwellian, Publ. Res. Inst. Math. Sci., 29 (1993), pp. 301-347.

[32] R. T. Glassey and W. A. Strauss, Asymptotic stability of the relativistic Maxwellian via fourteen moments, Transport Theory Statist. Phys., 24 (1995), pp. 657-678.

[33] R. T. Glassey and W. A. Strauss, On the derivatives of the collision map of relativistic particles, Transport Theory Statist. Phys., 20 (1991), pp. 55-68.

[34] Y. Guo, The Vlasov-Maxwell-Boltzmann system near Maxwellians, Invent. Math., 153 (2003), pp. 593-630.

[35] Y. GuO, The Vlasov-Poisson-Boltzmann system near Maxwellians, Comm. Pure. Appl. Math., 55 (2002), pp. 1104-1135.

[36] Y. Guo and R. Strain, Momentum regularity and stability of the relativistic Vlasov-MaxwellBoltzmann system, Comm. Math. Phys., 310 (2012), pp. 649-673.

[37] Y. Guo and R. Strain, Stability of the relativistic Maxwellian in a collisional plasma, Comm. Math. Phys., 251 (2004) pp. 263-320.

[38] T. Gustafsson, $L^{p}$ estimates for the nonlinear spatially homogenous Boltzmann equation, Arch. Ration. Mech. Anal., 92 (1986), pp. 23-57.

[39] T. Gustafsson, Global $L^{p}$ properties for the spatially homogeneous Boltzmann equation, Arch. Ration. Mech. Anal., 103 (1988), pp. 1-38.

[40] Z. JIANG, Global existence proof for relativistic Boltzmann equation with hard interactions, J. Stat. Phys., 130 (2008), pp. 535-544.

[41] H. Lee And A. Rendall, The Spatially Homogeneous Relativistic Boltzmann Equation with a Hard Potential, arXiv:1301.0106, 2013.

[42] P. L. Lions, Compactness in Boltzmann's equation via Fourier integral operators and applications. I, J. Math. Kyoto Univ., 34 (1994), pp. 391-427.

[43] P. L. Lions, Compactness in Boltzmann's equation via Fourier integral operators and applications. II, J. Math. Kyoto Univ., 34 (1994), pp. 429-461.

[44] P. L. Lions, Compactness in Boltzmann's equation via Fourier integral operators and applications. III, J. Math. Kyoto Univ., 34 (1994), pp. 539-584.

[45] X. Lu, Conservation of energy, entropy identity, and local stability for the spatially homogeneous Boltzmann equation, J. Stat. Phys., 96 (1999), pp. 765-796.

[46] S. Mischler And B. WennberG, On the spatially homogeneous Boltzmann equation, Ann. Inst. H. Poincarè Anal. Non Lineairè, 16 (1999), pp. 467-501.

[47] C. Моuнот, Rate of convergence to equilibrium for the spatially homogeneous Boltzmann equation with hard potentials, Comm. Math. Phys., 261 (2006), pp. 629-672.

[48] C. Mounot and C. Villani, Regularity theory for the spatially homogeneous Boltzmann equation with cut-off, Arch. Ration. Mech. Anal., 173 (2004), pp. 169-212.

[49] A. J. Povzner, On the Boltzmann equation in the kinetic theory of gases, Mat. Sb. (N.S.), 58 (1962), pp. 65-86.

[50] R. StRain, Asymptotic stability of the relativistic Boltzmann equation for the soft potentials, Comm. Math. Phys., 300 (2010), pp. 529-597.

[51] R. Strain, Coordinates in the relativistic Boltzmann theory, Kinet. Relat. Models, 4 (2011), pp. 345-359.

[52] R. STRAIN, Global Newtonian limit for the relativistic Boltzmann equation near vacuum, SIAM J. Math. Anal., 42 (2010), pp. 1568-1601.

[53] B. Wennberg, Entropy dissipation and moment production for the Boltzmann equation, J. Stat. Phys., 86 (1997), pp. 1053-1066.

[54] S. UKaI, On the existence of global solutions of a mixed problem for the nonlinear Boltzmann equation, Proc. Japan Acad. Ser. A, 50 (1974), pp. 173-221.

[55] C. Villani, A new class of weak solutions to the spatially homogeneous Boltzmann and Landau equations, Arch. Ration. Mech. Anal., 143 (1998), pp. 274-307.

[56] C. Villani, A Review of Mathematical Topics in Collisional Kinetic Theory, in Handbook of Mathematical Fluid Dynamics, vol. 1, North-Holland, Amsterdam, 2002, pp. 71-305. 